\title{
FROM PHYSICS TO BIOLOGY BY EXTENDING CRITICALITY AND SYMMETRY BREAKINGS: AN UPDATE ${ }^{1}$
}

\author{
Author(s) / Auteur(s) : \\ Giuseppe LONGO \\ Centre Cavaillès, République des Savoirs, \\ CNRS et Ecole Normale Supérieure, Paris \\ School of Medicine, Tufts University, Boston \\ http://www.di.ens.fr/users/longol \\ giuseppe.longo@ens.fr
}

\section{Maël MONTÉVIL}

Institut de recherche et d'Innovation, Centre Pompidou

Institut d'Histoire et de Philosophie des Sciences et des Techniques (IHPST)

Université Paris 1 Panthéon-Sorbonne

https://montevil.theobio.org/

mael.montevil@gmail.com

\begin{abstract}
Résumé :
Symmetries play a major role in physics, in particular since the work by E. Noether and H. Weyl in the first half of last century. Herein, we briefly review their role by recalling how symmetry changes allow to conceptually move from classical to relativistic and quantum physics. We then introduce our ongoing theoretical analysis in biology and show that symmetries play a radically different role in this discipline, when compared to those in current physics. By this comparison, we stress that symmetries must be understood in relation to conservation and stability properties, as represented in the theories. We posit that the dynamics of biological organisms, in their various levels of organization, are not "just" processes, but permanent (extended, in our terminology) critical transitions and, thus, symmetry changes. Within the limits of a relative structural stability (or interval of viability), qualitative variability is at the core of these transitions.
\end{abstract}

Keywords / Mots-clés :

symmetries, systems biology, critical transitions, levels of organization, hidden variables, coherent structures, downward causation.

\section{INTRODUCTION AND SUMMARY}

A synthetic understanding of the notion of organism requires drawing strong correlations between different levels of organization as well as between the global structure and the local phenomena within the organism. These issues should govern any systemic view on biology. Here, we sketch an approach in which the living state of matter is interpreted as a permanent "transition", conceived as an ongoing or extended and critical transition. A large amount of very relevant work pertaining to the Theories of Criticality in physics has been successfully applied to biology (see below). The mathematical core of these theories rests upon the idea that a "phase transition," which can be either critical or not, may be described as a point along the line where the intended control parameter runs. For example, the ferromagnetic / paramagnetic transition takes place for a precise value of the temperature, the Curie temperature. Mathematically, this is expressed by the "pointwise" value of this temperature, i.e., one mathematical point in this parameter's space. When the temperature decreases and passes through that point, the magnetic orientation organizes along one direction and magnetism appears. When the temperature increases through that point, disorder prevails and magnetism disappears. A (phase)

\footnotetext{
1 First published as: G. Longo and M. Montévil. 2011a. "From physics to biology by extending criticality and symmetry breakings." Systems Biology and Cancer, Progress in Biophysics and Molecular Biology 106 (2):340-347. issn: 0079-6107. doi:10.1016/j.pbiomolbio.2011.03.005
} 
transition is critical when some observables, or their first or second derivatives, diverge. This corresponds to the appearance of a "coherent structure", that is to say space and/or time correlations at all scales leading to a fractal geometry. As a result, at the transition point, the new physical object possesses a "global" structure. These ideas are relevant to the analysis of biological organisms.

In contrast to known critical transitions in physics, biological entities should not be analyzed just as transient over a point of a phase change; instead, they permanently sustain criticality over a non-zero interval and this with respect to many control parameters (time, temperature, pressure). This represents a crucial change of perspective. First, the mathematical tools used in physics for the analysis of criticality, i.e, the renormalization methods, essentially use the pointwise nature of the critical transitions. Secondly, symmetries and symmetry breakings radically change when enlarging the mathematical locus of criticality from one point to a non-zero interval. These symmetry changes make a key theoretical difference with respect to the few cases in physics where the transition seems extended (see footnote 10, below). Our approach may be seen as a move from physics to biology by an analysis of the radically different symmetries and symmetry breakings at play in their respective theoretical frames. Thus, we will mostly focus on physical vs biological criticality in terms of symmetries and then apply this method to the analysis of the difference between physical and biological "objects" as well as of physical vs biological "trajectories".

Living entities are not "just" processes, but something more: they are lasting, extended critical transitions, always transient toward a continually renewed structure. In general, physical processes do not change fundamental symmetries: to the contrary, they are mostly meant to preserve them. Typically, conservation properties (of energy, of momentum) are symmetries in the equations of movement. Critical transitions are an exception to the preservation of symmetries in physics; their "extension" radically changes the understanding of what biological processes are. This perspective also proposes a possible way of overcoming a key issue in the analysis of the complexity of the living state of matter. As for the construction of physico-mathematical or computational models, it is difficult to take the global structure of an organism into consideration, with its correlations between all levels of organization and in all lengths, including the many forms of integration and regulation. Thus, the complexity of the living unity is often modeled by the stacking of many but simple elementary processes. Typically, these formal systems deal with many observables and parameters. Since the framework is classical in a physical sense, these variables are local, i.e. they depend on pointwise values of the intended phase space. Instead, conceptual and mathematical dependencies in biology should be dealt with as "global" ones, where variables may depend on systemic or non-local effects. In physics, these dependencies are a relevant aspect of critical transitions, and they are even more so in biology, where criticality is extended.

\section{Hidden variables in biology?}

In classical and relativistic physics, once the suitable "phase space" and the equations that mathematically determine the system are given, the knowledge of the pointwise position-momentum of the intended object of analysis allows to describe in principle the subsequent dynamics. This is "in principle" since physical measurement, which is always approximated, may produce the phenomenon of deterministic unpredictability, in particular in the presence of non-linear mathematical determination ${ }^{2}$. Moreover, not all "forces" in the game may be known and there may be "hidden variables" (like the frictions along the trajectory of bouncing dice). Yet, these theories are deterministic and, once all pertinent variables and forces are assumed to be known, it is the epistemic lack of knowledge which yields classical randomness. Per se, a dice follows a "geodesic". This is a unique, optimal and "critical" path, completely determined by the Hamiltonian and may be computed as an optimum of a Lagrangian functional. ${ }^{3}$. This very beautiful paradigm, which may be summarized as the

\footnotetext{
2 More generally, unpredictability may appear when the dynamics is determined by an evolution function or equations that mathematically represent "rich" interactions. Non-linearity is a possible mathematical way to express them.

3 These are mathematical operators, that is, functions acting on functions that contain all known physical information concerning the energy state of the system.
} 
"geodesic principle", may be further grounded on symmetries by an analysis of conservation principles (see Bailly and Longo (2011) for a recent synthesis and references).

In order to compare this situation with other fields of physics and subsequently to biology, we refer to the pointwise or local nature of the mathematical variables. Cantorian (and Euclidian) points are limit conceptual constructions; that is, they are the limit of a physical access to space and time by an always approximated measurement, i.e., an "arbitrarily small" interval. Yet, their perfect theoretical "locality" makes all classical dynamics intelligible (in principle). So, if something is unknown, one expects that by adding enough observables and/or more variables with definite values at any given time, one could increase knowledge, since the values of these observables are intrinsic and independent of the context.

The situation is rather different in Quantum Mechanics. The simultaneous, perfect, pointwise knowledge of position and momentum (or energy and time) are, in principle, forbidden because indeterminacy is intrinsic to the theory. Moreover, suppose that two quanta interact and form one system and that they later separate in space. Then acquiring knowledge regarding an observable quantity by performing a measurement on one of these quanta produces an instantaneous knowledge of the value of the measurement made on the other, i.e., the two quanta are "entangled" (Einstein, Podolsky, and Rosen 1935). These features of the theory have several consequences: for instance, variables cannot always be associated to separated points and quantum randomness is intrinsic (under the form of Schrödinger equation, the "determination" gives the probability to obtain a value by measurement). Within this theoretical framework, quantum randomness differs from the classical one: two interacting dice which later separate obeying independent statistics, while the probability values of an observable of two previously interacting quanta are correlated. This is the so called "violation of Bell inequalities", which has been empirically verified repeatedly since the experiments described in Aspect, Grangier, and Roger (1982). Quantum entanglement requires considering some phenomena as being "non-local" and unseparable by any physical measurement ("non-separability").

Since the '30s, some have found this situation unsatisfactory and have searched for "hidden variables" like in the epistemic approach to randomness and determination of classical and relativistic physics. The idea is that these hidden variables corresponding to quantum mechanical observables have definite (pointwise/local) values at any given time, and that the values of those variables are intrinsic and independent of the device used to measure them. A robust result has instead shown that these assumptions contradict the fundamental fact that quantum mechanical observables need not be commutative (Kochen and Specker 1967). Moreover, even when assuming the existence of, or the need for, hidden variables, these would be "non-local" and thus, far from the pointwise/local dependence of set-theoretic variables.

The difference between the classical and quantum frameworks has the following consequence: quantum systems may have a proper systemic unity for at least two reasons. Conjugated observables (position and momentum) are "linked" by joint indetermination, and entangled quanta remain a "system", in the sense of their non-separability by measurement ${ }^{4}$.

Can this perspective help us in biology? On technical grounds, surely not, or rather not yet. Perhaps, "entangled molecular phenomena" or "tunnel effects ... in the brain" may clarify fundamental issues in the future. However, theoretical ideas in Quantum Mechanics may at least inspire our attempts in systems biology, in particular by considering the methodological role of symmetries and symmetry breakings in this area of physics.

A living organism is a system. And entanglement, non locality, non-separability, superposition, whatever these concepts may mean in biology, may present themselves both at each specific level of organization and in the interactions between levels of organization. Physiological interactions among molecules, cells, tissues, organs do not simply sum each other up: they are "entangled", "non-local", "non-separable" ... they are "superposed" (see examples described by Noble (2006; Soto, Sonnenschein, and Miquel 2008)). Thus, the theoretical and mathematical approaches to biology cannot be based only on a continual enrichment of "local" views: mathematical models cannot work just by

\footnotetext{
${ }^{4}$ Superposition should also be mentioned, see Silverman (2008).
} 
assuming the need for more and more variables (possibly hidden to the previous models). A global view of the system and of its symmetries is required, which requires, among other, specific analysis of measurement (Montévil 2019a). In this context, the differences in symmetries and their breakings will help in clarifying and facilitating the passage from physics to biology.

\section{SYMMETRY AND OBJECTIVATION IN PHYSICS}

In Physics, objectivity is obtained by the co-constitutive use of experiments and mathematized theories. So far, however, there is little mathematics for a "theory of the biological organisms" despite the large amount of data collected and of theories proposed within specific levels of organization. These results include the geometric analysis of the fractal structures of lungs, of vascular systems, of various plant organs, of networks of neural cells, of tumor shapes, to name but a few. To make further progress towards mathematizing theories in biology, in particular towards theories of the "living object" or of the organism as a system, it would help first to understand how such a feat was achieved in physics. Physical theories have very general characteristics in their constitution of objectivity, and in particular in their relationship with mathematics. In order to define space and time, as well as to describe physical objects, physicists ultimately use the notion of symmetry. Physical symmetries are the transformations that do not change the intended physical aspects of a system in a theory. As we shall see, they allow to define these aspects in a non-arbitrary way.

Galileo's theory provides a simple and historical example of this role of symmetries. For scholastic physics, the speed at which a body falls is proportional to the space traveled. Galileo instead proposed that it is proportional to the time of the fall and that it is independent of the nature (including the mass) of the empirical object considered (Galileo' law of gravitation). This idea together with the "principle of inertia" has been a starting point for the constitution of space and time in classical physics. More precisely, as a consequence of the analysis of inertia and gravitation, the geometry of space and time was later described by the Galilean group ${ }^{5}$.

A change of this symmetry group, for example by adopting the Poincare group ${ }^{6}$, can lead to a radically different physical perspective, that of special relativity involving massive conceptual and physical changes. The "principle of relativity" states that the fundamental laws of physics do not depend on the reference system; they are actually obtained as invariants with respect to the change of reference system. A specific speed (the speed of light in the void) appears in the equations of electromagnetism. Einstein modified Galileo's group in order to transform this speed into an invariant of mechanics, which turned time-simultaneity into a relative notion.

As a result of the role and implications of symmetries, most contemporary physical challenges lead to the search for the right symmetries and symmetry changes, such as the efforts to unify relativistic and quantum theories. In moving from physics to biology we suggest here to apply a similar approach (symmetry changes).

Since the 1920s, due to Noether's theorems, symmetries lead to the mathematical intelligibility of key physical invariant quantities. For example, symmetries by time translations are associated with energyconservation, and symmetries by space rotations are associated with the conservation of angular momentum. Thus, conservation laws and symmetries are in a profound mathematical relation. Consequently, the various properties that define an object (mass, charge, etc.) or its states (energy, momentum, angular momentum, etc.) are associated to specific symmetries which allow these quantities to be defined. Depending on the theory adopted, this conceptualization allowed to understand why certain quantities are conserved or not: for example, there is no local energy conservation in general relativity. This explicit reference to the theory adopted is required in order to produce "scientific

\footnotetext{
5 Symmetries form a set of transformations that have a group structure; that is, two symmetries applied successively yield a symmetry and a symmetry can be inverted. Galileo's group is the group of transformations that allows to transform a Galilean space-time reference system into another. It is interesting to notice that Galileo measured time by heartbeat, a biological rhythm; the subsequent theoretical and more "physical" measurement of time were precisely provided by classical mechanics, his invention.

6 The symmetry group of a Euclidean space is the Euclidean group of automorphisms, while Poincaré's group corresponds to the automorphisms defining Minkowski's spaces.
} 
objectivity", independently of the arbitrary choices made by the observer, such as, the choice of time origin, the unit of measurement, etc, but relatively to the intended theory. Thus, we say that symmetries provide "objective determinations" in physics (Bailly and Longo 2011).

The symmetries that define physical properties allow us to understand the physical object as generic, which means that any two objects that have the same properties can be considered as physically identical; in a sense, they are symmetric or invariant (interchangeable) in experiments and in pertinent mathematical framework (typically, the equations describing movement). For example, for Galileo, all objects behave the same way in the case of free fall, regardless of their nature. Moreover, symmetries allow the use of the geodesic principle, whereby the local determination of trajectories leads to the determination of the full trajectory of physical objects through conservation laws. For example, the local conservation of the "tangent" (the momentum) of trajectories, typically yields the global "optimal" behavior of the moving object; that is, it goes along a geodesic. Thus, in classical or relativistic mechanics, a trajectory is unique and fully deterministic (formally determined). In quantum mechanics the evolution of the state or wave function (roughly, a probability distribution) is fully deterministic as well - and determined by Schrödinger's equation - while measurement follows this probability distribution (and here appears the indeterministic nature of quantum mechanics). In conclusion, by symmetries, the trajectory of a generic classical or quantum physical "object" corresponds to a critical path: physical trajectories are specific.

To better understand the problem of general mathematical theorizing in biology, let's further analyze how, in physics, a concrete problem is turned into robust models and mathematics. To begin with, physicists try to choose the right theoretical framework and the relevant physical quantities (properties and states) which are constituted by proper symmetries. As a result, typically, a mathematical framework is obtained, where one can consider a generic object; in classical mechanics, a pointwise object of mass $m$, velocity $v$ and position $x$, where these variables are generic - a pleonasm. Now, a generic object will follow a specific trajectory determined by its invariants obtained by calculus. A measurement is then made on the experimental object to determine the quantities necessary to specify where this object is in this mathematical framework, namely, what is its mass, initial position and speed. And finally, what specific trajectory will the object follow ... at least approximately. In classical or relativistic physics, to a specific measurement will correspond generic objects localized near the measurement due to the limited precision of this measurement. This value may have, in principle, an arbitrary high precision. In quantum mechanics, as we recalled above, the equational determination (Schroedinger's equation) yields the dynamics of a probability law ${ }^{7}$.

In classical dynamics, we face a well-known problem: the specific trajectories corresponding to a measurement can either stay close or diverge very rapidly. The linear situation corresponds to the first case, whereas the second situation is called "sensitive to initial conditions" (or chaotic, according to various definitions). Note that even the latter situation leads to the definition of new invariants associated to the dynamics: in other words, the attractors that have a precise geometrical structure. In both cases, these trajectories have robust properties with respect to the measurement. In quantum physics, the situation is more complex because the measurement is not deterministic. Yet, when approximations on the state function are performed, it leads to usually stable. robust statistics. In all cases, "robust" means invariant or approximately invariant in a definite mathematical sense, as concerns the measurement of states and properties of generic objects along specific trajectories. Thus, we can finally say that generic objects, which lead to a specific measurement, behave in the same way or approximately so. Notice that this property of robustness, allowed by the genericity of the object, is mandatory for the whole framework to be relevant. We insist that both genericity for objects and specificity for trajectories (geodesics) depend mathematically on symmetries.

In conclusion, in the broadest sense, symmetries are at the foundation of physics, allowing us to objectivize space and time, and constitute objects and trajectories. In their genericity, these objects follow specific trajectories associated with invariants that are robust with respect to measurement.

\footnotetext{
7 In quantum physics, "objects" do not follow trajectories in ordinary space-time, but they do it in a suitable, very abstract space, a Hilbert space (a space of mathematical functions); what "evolves" is a probability distribution.
} 


\section{SYMMETRY BREAKINGS AND CRITICALITY IN PHYSICS}

The physics of criticality is a relatively novel discipline that analyzes, typically by the renormalization techniques, some peculiar phase transitions, i.e., state changes (see Toulouse, Pfeuty, and Barton (1977; Binney et al. 1992)). This theoretical framework has also been applied to a possible understanding of life phenomena (see for example, Bak, Tang, and Wiesenfeld (1988; Jensen 1998), as for "self-organized criticality"; or, Kauffman (1993), as for criticality in networks). We will next move towards biology through a different insight into the symmetries in criticality.

Since symmetries are at the core of the definition of the physical objects by their properties and states, a symmetry change (that is, the breaking of some symmetries and the formation of new ones) means a qualitative change of the object considered, or a change of physical object, understood as co-constituted by theory and empiricity. For example, a research program in cosmology is to consider a single force to have existed in the universe right after the big bang. Then, the four fundamental forces would have appeared by successive symmetry breakings, whereby some transformations, which were symmetries, did not preserve the object invariance anymore. In other words, with the cooling of the universe, the system moved to a smaller symmetry group. Closer to the scale of biology, materials like water or iron have different properties in different situations. Depending on the temperature and pressure, water may be a solid, a liquid, or a gas. When liquid, there is no privileged direction (the system is isotropic, that is to say symmetric by rotations), whereas ice has a crystalline structure with spatially periodic patterns. This structure implies that the system is no longer symmetric by continuous rotations: it has a few privileged directions determined by its crystalline structure and a smaller symmetry group. Similarly, iron can have paramagnetic behavior (the system is not spontaneously magnetic) or ferromagnetic behavior (it is). In most cases, one can distinguish a more disordered phase at high temperature, where entropy dominates, and a more ordered phase, where energy dominates. These situations can be characterized by an order parameter which is 0 in the disordered phase and different from 0 in the ordered phase ${ }^{8}$.

Now, in physics, the change of state, or phase transition, occurs always mathematically at a point of the parameters' space. This point, called the critical point, is intuitively associated with a sudden change of behaviour due to a change of symmetry, and ultimately to singularities of the state functions (for example, the order parameter is non-analytical because it goes from a constant 0 to a finite quantity, by a finite change). More technically, the critical point represents a singularity in the partition function describing the system ${ }^{9}$. In the case of iron's paramagnetic-ferromagnetic transition, this allows to deduce the divergence of some physical observables, such as magnetic susceptibility. It should be remembered that this notion of singularity, which is associated with infinite quantities at the critical point, is a core notion for physical criticality.

This peculiar situation leads to a very characteristic behaviour at the critical point (Jensen 1998):

1. Correlation lengths tend to infinity, and follow a power law, as for continuous phase transitions (i.e., for a vector $x$ and an observable $N$, if we note by $\langle\cdot\rangle_{r}$ the average over point $r$ in space, then $<N(r+x) N(r)>_{r}-<N(r)>_{r}^{2} \sim\|x\|^{\alpha}$. This is associated with fluctuations at all scales leading in particular to the failure of mean field approaches. Following this approach, the value of an observable at a point is given by the mean value in its neighbourhood or, more precisely, its mathematical distribution is uniform.

2. Critical slow down: the time of return to equilibrium of the system after a perturbation tends to infinity (Suzuki, Kaneko, and Takesue 1982; Tredicce et al. 2004; Longo and Montévil 2014a).

3. Scale invariance: the system has the same behavior at each scale. This property leads to fractal geometry and means that the system has a specific symmetry (scale invariance itself).

4. The determination of the system is global and no longer local.

\footnotetext{
8 Here, order means low entropy (or less symmetries) and disorder means high entropy (and more symmetries, when symmetries are computed in terms of "microstates").

9 This function is non-analytical at the critical point, which means that the usual Taylor expansions, linearizations or higher order approximations do not actually provide an increasing approximation.
} 
These properties are the key motivations for the biological interest of this part of physics. The global "coherence structure" that is often formed at critical transitions provides a possible understanding, or at least, an analogy for the unity of an organism (in current terminology, its "global determination or causation"). Also, power laws, so frequent in biology, are ubiquitous in critical phenomena. They are mathematically well-behaved functions (e. g. $f(x)=x^{\alpha}$ ) with respect to the change of scale [typically, $\lambda$ is the scale change in $f(\lambda x)=\lambda^{\alpha} f(x)=\lambda^{\alpha} x^{\alpha}$, a power law in $\alpha$, and they yield scale symmetries. In our example, scale change just multiplies the function $f$ by a constant $\lambda^{\alpha}$. Now, a power law depends on a quantity without physical dimension ( $\alpha$ in the notation above). These quantities involved in critical transitions are called critical exponents and describe how the change of scale occurs. In our terminology, they describe the properties due to the objective determination of a phase transition because they are the invariants associated with the scale symmetry.

Specific analytical methods, called renormalization methods, are used to find these quantities (Delamotte 2004; Longo, Montévil, and Pocheville 2012). These methods consist in analyzing how scale changes transform a model representing the system, and this analysis is made "asymptotically" toward large scales. One may deduce the critical exponents from the mathematical operator representing the change of scale. The key point is that a variety of models ultimately lead to the same quantities, which means that they have the same behavior at macroscopic scales. Thus, they can be grouped in so-called universality classes. This analytical feature is confirmed empirically, both by the robustness of its results for a given critical point and more stunningly by the fact that very different physical systems happen to undergo the same sort of phase transitions; that is, they are associated with the same critical exponents, thus with the same symmetries. Finally, there exist fluctuations at all scales, which means, in particular, that small perturbations can lead to very large fluctuations.

To conclude, the transition through a specific point of the parameters' space, i.e., a transition between two very different kinds of behavior is associated in physics to a change of symmetries. At this point, the system has very peculiar properties and symmetries. Symmetries by dilation (by a coefficient $\lambda$ as above) yield a scale invariance. This latter invariance is associated to a global determination of the system and the formation of a "structure of coherence". As observed above, this allows to describe a global determination of local phenomena and a unity that by-passes the idea of understanding the global complexity as the sum of many local behaviors by adding more and more local, possibly hidden, variables. For some physical phenomena this theoretical framework presents peculiar and very relevant forms of "systemic unity" (Longo, Montévil, and Pocheville 2012).

\section{SYMMETRY BREAKING AND THE BIOLOGICAL OBJECT: EXTENDED CRITICALITY}

We have presented a picture of the situation in physics, but what about biology? We need to propose one or several specific frameworks relevant to the unity and coherence of biological entities, because, to our knowledge, there are no formalized theories of the "organism" (Noble 2008; Soto et al. 2016). To do so, it may be worthwhile to look at the symmetries which may be involved in biological theorizing. Here, the concept of symmetry is used in a more fundamental context than when used, for example, for "bauplans", the latter being the main biological research subjet where the concept is explicitly applied. In physics, one mostly deals with fundamental or theoretical symmetries as typically given by the equations. For example, the already mentioned fundamental principle of energy conservation corresponds to a time translation symmetry in the equations of movement. This use of symmetries also justifies the soundness of empirical results: Galilean inertia is a special case of conservation of energy and it may be empirically verified. In biology, as in any science, a missing analysis of invariants may give unreliable results and data. The current reproducibility crisis may be analyzed in this context (Begley and Ioannidis 2014; Montévil 2019a). For example, early measurements of membrane surfaces gave very different results, since their measure is not a scale invariant property: as in fractal structures, 
it depends on the scale of observation ${ }^{10}$. In other words, in physics, both the generality of equations and the very objectivity of measures depend on theoretical symmetries and their breakings, such as scale invariants and scale dependencies.

As mentioned above, critical transitions in physics are mathematically analyzed as isolated points ${ }^{11}$. In our approach to biological processes as "extended critical transitions", "extended" means that every point of the evolution/development space is near a critical point. More technically, the critical points form a dense ${ }^{12}$ subset of the multidimensional space of viability for the biological process. Thus, criticality is extended to the space of all pertinent parameters and observables (or phase space), within the limits of viability (tolerated temperature, pressure and time range, or whatever other parameter, say for a given animal), see Bailly, Gaill, and Mosseri (1993; Bailly and Longo 2008, 2011). In terms of symmetries, such a situation implies that biological objects (cells, multicellular organisms, species) are always close to a transition between different symmetry groups; that is, they are in transition between different phases, according to the language of condensed matter ${ }^{13}$. These phases swiftly shift between different critical points and between different physical determinations through symmetry changes. Several work begin to explore this notion mathematically (Sarti, Citti, and Piotrowski 2019; Montévil 2019).

Our perspective provides an approach concerning the mathematical nature of biological objects as a limit or asymptotic case of physical states: the latter may yield the dense structure we attribute to extended criticality only by an asymptotic accumulation of critical points in a non-trivial interval of viability - a situation not considered by current physical theories. In a sense, it is the very principles grounding physical theories that we are modifying through an "actual" limit. Thus, a biological object is mathematically and fundamentally different from a physical object because it may be characterized in terms of partial but continual changes of symmetry within an interval of viability, as an extended locus of critical transitions. In particular, this mathematical view of "partial preservation through symmetry changes" is a way to characterize the joint dynamics of structural stability and variability proper to life (Montévil 2019, n.d.). We thus consider this characterization as a tool for the mathematical intelligibility of fundamental biological principles: the global/structural stability is crucially associated with variability.

A first consequence of these permanent symmetry changes is that there are very few invariants in biology. Mathematically, invariants depend on stable symmetries. Structural stability in biology, thus, should be understood more in terms of correlations of symmetries within an interval of the extended critical transition, rather than on their identical preservation. It is clear that the bauplan and a few more properties are more preserved than others. Yet, in biology, theoretical invariants are continually broken by these symmetry changes. A biological object (a cell, a multicellular organism, a species) continually changes symmetries, with respect to all control parameters, including time. Each mitosis is a symmetry change because the two new cells are not identical. This variability, under the mathematical form of symmetry breaking and constitution of new symmetries, is essential both for evolution and embryogenesis. The interval of criticality is then the "space of viability" or locus of the possible structural stability.

The changes of symmetries in the dense interval of criticality, which provide a mathematical understanding of biological variability, are a major challenge for theorizing. As a matter of fact, we are

\footnotetext{
${ }^{10}$ In Weibel (1994), another "historical" example is given as for the different results that are obtained according to different experimental scales (microscope magnifications). One team evaluated the surface density of the liver's endoplasmic reticulum at 5.7 the other at $10.9($ !).

11 The Kosterlitz-Thouless transition in statistical physics presents a marginally critical interval; that is, it is a limit case between critical and not critical. It presents correlations at all scales, as critical features, but with no symmetry changes. Thus, this particular situation is not a counter-example to our statement (the essentially pointwise nature of the proper physical transitions), in view of a lack of symmetry changes that are essential to our notion of extended criticality, see also Lovecchio et al. (2012).

${ }^{12}$ Here, dense means that for every small volume of the intended phase space being considered, there is a critical point in such volume.

${ }^{13}$ The dense set of symmetry groups may be potentially infinite, but, of course, an organism (or a species) explores only finitely many of them in its life span, and only viable ones.
} 
accustomed to the theoretical stability warranted by the mathematical invariants at the core of physics. These invariants are the result of symmetries in the mathematical (equational) determination of the physical object. This lack of invariants and symmetries corresponds to the difficulties in finding equational determinations in biology ${ }^{14}$.

As a further consequence of our approach, phylogenetic or ontogenetic trajectories cannot be defined by the geodesic principle, since they are not determined by invariants and their associated symmetries. These latter are continually changing in a relatively minor but extended way.

Biology may be considered to be in an opposite situation with respect to physics: in contrast to physics, in biology, trajectories are generic whereas objects are specific (Bailly and Longo 2011). That is, a rat, a monkey or an elephant are the specific results of possible (generic) evolutionary trajectories of a common mammal ancestor - or each of these individuals is specific. They respectively are the result of a unique constitutive history, yet a possible or generic one (Bailly, Gaill, and Mosseri 1993; Bailly and Longo 2011).

The evolutionary or ontogenetic trajectory of a cell, a multicellular organism or a species is just a possible or compatible path within the ecosystem. The genericity of the biological trajectories implies that, in contrast to what is common in physics, we cannot mathematically and a priori determine the ontogenetic and phylogenetic trajectory of a living entity be it an individual or a species. In other words, in biology, we should consider generic trajectories (or possible paths) whose only constraints are to remain compatible with the survival of the intended biological system. Thus, phylogenesis and embryogenesis are possible, or at least not impossible paths subject to various constraints, including of course the inherited structure of the DNA, of the cell and the ecosystem. The specificity of the biological object, instead, is the result of critical points and of symmetry changes of the system considered along its past history, both evolutionary and ontogenetic (Montévil et al. 2016; Longo 2018). These constitute the specific "properties" of this object, which allow to define it. A rat, a monkey or an elephant or their species are specific and cannot be interchanged either as individuals nor as species. A living entity is the result of its history and cannot be defined "generically" in terms of invariants and symmetries as it is done for physical objects and actual empirical practices cannot avoid this fact (Montévil 2019a).

This situation has a particular meaning when we consider time translation and time reversal symmetries. In physics, time symmetries correspond to the maintaining of the system's invariant quantities that define the geodesics, as for example, conservation of energy. In biology both symmetries are broken. In particular, evolutionary and ontogenetic paths are both irreversible and non-iteratable; there is no way to identically "rewind" nor "restart" evolution or ontogenesis. This corresponds to the breaking of time translation and reversal symmetries. In particular, this lack of time symmetries is associated with the process of individuation, understood here as the specificity of cells, organisms and species (as much as this latter notion is well defined). It is crucial to understand that time plays a key role in this framework, since the history of all the changes in symmetry are not reducible to a specific trajectory in a given space of the dynamics.

The sequence of symmetry changes defines the historical contingency of a living object's phylogenetic or ontogenetic trajectory.

Biological processes are more "history based" than physical processes. Usual physical processes preserve invariants, whereas extended critical transitions are a permanent reconstruction of organization and symmetries, i.e., of invariants. This situation also points to a lack of symmetry by permutation. For example, even in a clonal population of bacteria, different bacteria are not generic, because they are in general not interchangeable, i.e., they cannot be permuted. This allows to understand biological variability in a deeper way than the usual Gaussian (or combination of Gaussians) as random distribution of a set of observables. Now, let us consider organs (and organelles). Some organs have a functional role that can be expressed in a physical framework, particularly as far as energy transfer is concerned.

\footnotetext{
${ }^{14}$ In a rather naive way, some say this by observing that any (mathematized) theory in biology has a "counterexample". This instability of the determination goes together with the "structural stability" of biological entities. This is largely due to the stabilizing role of integration and regulation effects between different levels of organization. The mathematics of extended criticality and of variants of the renormalization methods are yet to be developed.
} 
This functional role can lead to constraints on the variability of the cells that constitute the organ, while the same could be said for individual organisms in populations. At least for certains aspects of their behaviour and on average, these constraints make cells behave symmetrically. In other words, those cells behave, in part and approximately, like generic objects with specific trajectory (geodesics). For these aspects, they may be interchangeable, like physical objects, to an extent.

The simple case of cells secreting a protein such as erythropoietin (EPO) under specific conditions indicate that on average, a sufficient amount of the protein must be produced, independently of the individual contribution of each cell (which become "relatively" generic). Since the result of these cells' production is additive (linear), its regulation does not need to be sharp. Even if some cells do not produce EPO there is no functional problem as long as a sufficient quantity of this protein is secreted at the tissue level. However, when cells contribute to a non-linear framework as part of an organ, the regulation may need to be sharper. This is the case, for example, for neuronal networks or for cell proliferation where non-linear effects may be very important. In the latter case, regulation by the tissue and the organism seems to hold back pathological developments, like cancer, see Sonnenschein and Soto (1999). This point of view can possibly be generalized in order to understand the robustness of development.

The role of physical processes in shaping organs is crucial; for example, exchanges of energy (or matter) force/determine the optimal (geodesic) fractal structure of lungs and vascular systems. Organs in an organism may even be replaced by man-made artifacts (as for kidneys, heart, limbs, etc.). As biological entities, organisms and even cells are specific or, at most, weakly generic given that they can be interchanged only within a given population or tissue and occasionally. In general, they are not generic, and by their specificity they cannot be replaced by an artifact - structurally.

In summary, in critical transitions one may consider variables depending on global processes because of the formation of coherent structures. For example, there may be functional dependencies on a network of interactions, which cannot be split into a sum of many local dependencies (local variables). Thus, the search for more variables would not take into account this fundamental property of biological systems, considered as extended critical transitions. Moreover, symmetries in physics allow to define generic objects which follow specific trajectories (the latter allowing to find invariants in terms of symmetries, which are robust regarding measurement). On the contrary, in biology, the continual symmetry changes lead to generic trajectories that remain compatible with the survival of the system. The generic/specific duality with respect to physics helped us understand this key issue, in relation to extended criticality which is a form of "relatively stable instability." In other words, this is stability under changes of symmetries in an interval of viability. In a sense, the biological object is also defined by its symmetries but in a very different way: it is the specific result of a history, where its dynamics is punctuated by symmetry changes. This makes it "historical" and contingent (Montévil et al. 2016; Longo 2018; Montévil 2019b).

\section{ADDITIONAL CHARACTERISTICS OF EXTENDED CRITICALITY}

In physics, criticality implies more than a pointwise symmetry change; that is, it requires a change on a mathematical point, as it leads to peculiar behaviors that are relevant to biology. The first of these properties is that criticality implies a global determination, instead of a simply local one. More precisely, the singularities involved in criticality lead to a change of the level of organization in a very strong sense. Also in physics, in view of the mathematical divergence of some observables, the singularities break the ability of the "down level" to provide a causal account of the phenomena and they lead to the need for a "top level" to overcome this difficulty. In mathematical physics, this upper level can be found in the renormalization operator (it is the abstract level of changing scale). In biology, instead, the upper level is the functional unity of an organism. As a result, the existence of different levels of organization is a component of our notion of extended critical transition. "Downward causation" may find the right frame of analysis in this theoretical context.

The permanent reconstruction of these levels of organization is mathematically represented by the density of the critical points and by the continual change of determination (symmetry change) in the passage between these points within the interval of extended criticality. 
The second property is the presence of power laws which seem to be ubiquitous in biology. They appear regularly especially when regulation is concerned, such as in cardiac rhythms (Makowiec et al. 2006; Pikkujamsa et al. 1999), blood cell number regulation (Perazzo et al. 2000), blood pressure (Wagner, Nafz, and Persson 1996), in brain activities (Werner 2007), sensory cells (Camalet et al. 2000), mitochondrial networks (Aon, Cortassa, and O'Rourke 2004), in ecology (Sole et al. 1999), collective behaviors (Mora and Bialek 2011) and gene network dynamics and structure (Shmulevich, Kauffman, and Aldana 2005; Nykter et al. 2008; Krotov et al. 2014; Valverde et al. 2015).

Extended critical transitions also concern the relevant lengths of local and global exchanges, the temporalities mobilized for such exchanges and biological rhythms. To summarize, the extended critical situation has at least the following characteristics (Bailly and Longo 2008, 2011):

1. A spatial volume enclosed within a semi-permeable membrane;

2. Correlation lengths of the order of magnitude of the greatest length of the above referred volume;

3. A metabolic activity that is far from equilibrium and irreversible, involving exchanges of energy, of matter and of entropy with the environment, as well as the production of entropy due to all these irreversible processes, see Bailly and Longo (2009; Longo and Montévil 2012);

4. An anatomo-functional structuralization into levels of organization that can be autonomous but also coupled to each other. They are "entangled" in the sense defined by Bailly and Longo (2009; Soto, Sonnenschein, and Miquel 2008). These levels are distinguished by the existence of fractal geometries (membranous or arborescent), where the fractal geometries can be considered as the trace (or a "model") of effective passages to the infinite limit of an intensive magnitude of the system (for example, local exchanges of energy ${ }^{15}$, Longo, Montévil, and Pocheville (2012)). The different levels of organization induce, and are a consequence of, the alternation of "organs" and "organisms", such as organelles in cells, which, in turn, make up the organs in multicellular organisms. Organisms stay in an extended critical transition, while organs are partially "optimally shaped" by the exchange of physical energy and matter. For example, fractal geometries essentially manifest in organs that are also the privileged loci of endogenous rhythms (see below). Correlation lengths are manifested both in and between these levels ${ }^{16}$. Likewise, the various biological "clocks" are coupled, and in some cases even synchronized, within and between these levels. Last, this space-time organization enables the interdependency between the parts of cells and organisms, a fundamental topic in theoretical biology (Mossio, Montévil, and Longo 2016). We described symmetries which are met for a time as constraints. Constraints canalize processes of transformation and we posited that constraints of an organism collectively maintain each other, leading to a circularity called closure of constraints (Montévil and Mossio 2015).

With the purpose of providing biological temporality with a structuring of the mathematical type, we will consider two other aspects as being specific to extended criticality.

- The two-dimensionality of time, proposed in (Bailly, Longo, and Montévil 2011):

1. One dimension is classical and is parametrized according to the line of real numbers limited by fertilization on one side, and death on the other. This dimension is linked to the biophysicochemical evolution of the organism in relation to an environment.

2. The other dimension is compactified, i. e. it is parametrized on a circle. This second dimension is linked to the organism's endogenous physiological rhythm that is manifested

\footnotetext{
15 The fractal dimension of some organs may be calculated by optimizing the purely physical exchanges within the intended topological dimension (for example, the maximization, within a volume, of surfaces for lungs, or of volumes for the vascular system, West, Brown, and Enquist (1997; Longo and Montévil 2014b)), and it may be subjected to constraints in terms of stericity and homogeneity, as in the cases mentioned (lung, vascular system, kidney, etc).

16 The term "entanglement" in Soto, Sonnenschein, and Miquel (2008) does not correspond, of course, to the physical meaning of "quantum entanglement" as expressed by Schrödinger's treatment of the state function and the inseparability of quantum measure, yet it may be appropriate because there is no way to isolate one of the organs mentioned above (e.g. put a brain in a flowerpot) and perform any reasonable physiological measure on it.
} 
through numeric quantities without dimension such as the mean total number of heartbeats and respirations during the lifetime of mammals. These are the interesting interspecific invariants and they are "pure" numbers, not frequencies (they have no dimension; they are the "total number of ..."). They become frequencies (with the inverse of time as a dimension), according to the average lifespan. The extra dimension is needed exactly because the invariant phenomenon is not defined by a period which has the dimension of time, but by this new invariant observable. For example, on average, the identical (invariant) number of total heartbeats give different frequencies according to the different lifespans of an elephant or of a mouse.

Moreover, the temporality of extended criticality involve protention (i.e. pre-conscious expectation) and retention (i. e. pre-conscious memory) (Longo and Montévil 2011), which seems to lead to a breaking of information conservation in cognition.

- The confinement within a volume of a parameter space (such as temperature, pressure, etc) of $n$ dimensions of which ${ }^{3}$ are spatial and ${ }^{2}$ temporal and whose measure is different from

0 (see above).

\section{CONCLUSION}

Since ancient Greece (Archimedes' principle on equilibria) up to Relativity Theory (and Noether's and Weyl's work) and Quantum Mechanics (from Weyl's groups to the time-charge-parity symmetry), symmetries have provided a unified view of the principles of theoretical intelligibility in physics. We claimed here that some major challenges for the proposal of mathematical and theoretical ideas in biology depend, in principle, on the very different roles that symmetries play in biology when compared to physics. The unifying theoretical framework in biology is neither associated to invariants nor to transformations preserving invariants like in (mathematical/theoretical) physics. It focuses, instead, on the permanent change of symmetries that per se modify the analysis of the internal and external processes of life, both in ontogenesis and evolution.

In a sense, variability may be considered as the main invariant of the living state of matter (Montévil et al. 2016). In order to explain it, we proposed to consider the role played by local and global symmetry changes along extended critical transitions. In extended criticality, dynamically changing coherent structures as global entities provide an understanding of variability within a global, extended stability. The coherent structure of critical phenomena also justifies the use of variables depending on non-local effects. Thus, an explicitly systemic approach may help in avoiding the accumulation of models and previously hidden variables. In conclusion, the notion of extended criticality provides a conceptual framework, to be further mathematized, where the dynamics of symmetries and symmetry breakings provide a new, crucial role for symmetries in biology with respect to physics.

\section{Aknowledgement:}

We warmly thank the editors of the first published version of this paper for several and very close preliminary revisions of this conceptually difficult text.

\section{REFERENCES}

Aon, M. A., S. Cortassa \& B. O'Rourke. (2004). "Percolation and Criticality in a Mitochondrial Network." Proceedings of the National Academy of Sciences 101 (13): 4447. 10.1073/pnas.0307156101.

Aspect, A., P. Grangier \& G. Roger (1982). "Experimental Realization of Einstein-Podolsky-RosenBohm Gedankenexperiment: A New Violation of Bell's Inequalities." Physical Review Letters 49 (2): 91-94. 10.1103/PhysRevLett.49.91.

Bailly, F., F. Gaill \& R. Mosseri. (1993). "Orgons and Biolons in Theoretical Biology: Phenomenological Analysis and Quantum Analogies." Acta Biotheoretica 41 (1-2): 3-11. 10.1007/BF00712769. 
Bailly, F. \& G. Longo. (2008). "Extended Critical Situations: The Physical Singularity of Life Phenomena." Journal of Biological Systems 16 (2): 309. 10.1142/S0218339008002514.

Bailly, F. \& G. Longo. (2009). "Biological Organization and Anti-Entropy.” Journal of Biological Systems 17 (1): 63-96. 10.1142/S0218339009002715.

Bailly, F. \& G. Longo. (2011). Mathematics and the Natural Sciences; the Physical Singularity of Life. London: Imperial College Press. 10.1142/p774.

Bailly, F., G. Longo \& M. Montévil. (2011). “A 2-Dimensional Geometry for Biological Time.” Progress in Biophysics and Molecular Biology 106 (3): 474-84. 10.1016/j.pbiomolbio.2011.02.001.

Bak, P., C. Tang \& K. Wiesenfeld. (1988). "Self-Organized Criticality.” Physical Review A 38 (1): 364 74. 10.1103/PhysRevA.38.364.

Begley, C. G. \& J. P. A. Ioannidis. (2014). "Reproducibility in Science." Circulation Research 116 (1): 116-26. 10.1161/CIRCRESAHA.114.303819.

Binney, J., N. J. Dowrick, A. J. Fisher \& M. E. J. Newman. (1992). The Theory of Critical Phenomena: An Introduction to the Renormalization Group. Oxford U. P.

Camalet, S., T. Duke, F. Julicher \& J. Prost. (2000). "Auditory Sensitivity Provided by Self-Tuned Critical Oscillations of Hair Cells." Proceedings of the National Academy of Sciences, 3183-8. 10.1073/pnas.97.7.3183.

Delamotte, B. (2004). “A Hint of Renormalization.” American Journal of Physics 72: 170. $10.1119 / 1.1624112$.

Einstein, A., B. Podolsky \& N. Rosen. (1935). "Can Quantum-Mechanical Description of Physical Reality Be Considered Complete?” Physical Review 47 (10): 777-80. 10.1103/PhysRev.47.777.

Jensen, H. J. (1998). Self-Organized Criticality, Emergent Complex Behavior in Physical and Biological Systems. Cambridge lectures in Physics.

Kauffman, Stuart A. (1993). The Origins of Order: Self Organization and Selection in Evolution. Oxford university press.

Kochen, S. \& E. P. Specker. (1967). "The Problem of Hidden Variables in Quantum Mechanics." Journal of Mathematics and Mechanics 17: 59-87. 10.1103/RevModPhys.38.447.

Krotov, D., J. O. Dubuis, T. Gregor \& W. Bialek. (2014). "Morphogenesis at Criticality." Proceedings of the National Academy of Sciences 111 (10): 3683-8. 10.1073/pnas.1324186111.

Longo, G. (2018). "How Future Depends on Past and Rare Events in Systems of Life." Foundations of Science 23 (3): 443-74. 10.1007/s10699-017-9535-x.

Longo, G. \& M. Montévil. (2011). "Protention and Retention in Biological Systems." Theory in Biosciences 130 (2): 107-17. 10.1007/s12064-010-0116-6.

Longo, G. \& M. Montévil. (2012). "Randomness Increases Order in Biological Evolution." In Computation, Physics and Beyond, edited by M. Dinneen, B. Khoussainov \& A. Nies, 7160:289-308. Lecture Notes in Computer Science. Springer Berlin / Heidelberg. 10.1007/978-3-642-27654-5_22.

Longo, G. \& M. Montévil. (2014a). "Protention and Retention in Biological Systems." In Perspectives on Organisms, 99-119. Lecture Notes in Morphogenesis. Springer Berlin Heidelberg. 10.1007/978-3642-35938-5_4.

Longo, G. \& M. Montévil.. (2014b). "Scaling and Scale Symmetries in Biological Systems." In Perspectives on Organisms, 23-73. Lecture Notes in Morphogenesis. Springer Berlin Heidelberg. 10.1007/978-3-642-35938-5_2.

Longo, G., M. Montévil \& A. Pocheville. (2012). "From Bottom-up Approaches to Levels of Organization and Extended Critical Transitions." Frontiers in Physiology 3 (232). 10.3389/fphys.2012.00232.

Lovecchio, E., P. Allegrini, E. Geneston, B. J. West \& P. Grigolini. (2012). "From Self-Organized to Extended Criticality." Frontiers in Physiology 3 (98). 10.3389/fphys.2012.00098. 
Makowiec, D., R. Galažska, A. Dudkowska, A. Rynkiewicz \& M. Zwierz. (2006). "Long-Range Dependencies in Heart Rate Signals-Revisited." Physica A: Statistical Mechanics and Its Applications 369 (2): 632-44. 10.1016/j.physa.2006.02.038.

Montévil, M. (2019). “Which First Principles for Mathematical Modelling in Biology?” Rendiconti Di $\begin{array}{llllll}\text { Matematica E Delle } & \text { Sue Applicazioni } 40 & \text { (3-4): }\end{array}$ http://www1.mat.uniroma1.it/ricerca/rendiconti/40_3-4_(2019)_177-189.html.

Montévil, M. (2019a). "Measurement in Biology Is Methodized by Theory." Biology \& Philosophy 34 (3): 35. 10.1007/s10539-019-9687-x.

Montévil, M. (2019b). "Possibility Spaces and the Notion of Novelty: From Music to Biology." Synthese 196 (11): 4555-81. 10.1007/s11229-017-1668-5.

Montévil, M. (n.d.) "Entropies and the Anthropocene Crisis." AI and Society.

Montévil, M. \& M. Mossio. (2015). "Biological Organisation as Closure of Constraints." Journal of Theoretical Biology 372: 179-91. 10.1016/j.jtbi.2015.02.029.

Montévil, M., M. Mossio, A. Pocheville \& G. Longo. (2016). "Theoretical Principles for Biology: Variation." Progress in Biophysics and Molecular Biology 122 (1): 36-50. 10.1016/j.pbiomolbio.2016.08.005.

Mora, T. \& W. Bialek. (2011). "Are Biological Systems Poised at Criticality?” Journal of Statistical Physics 144 (2): 268-302. 10.1007/s10955-011-0229-4.

Mossio, M., M. Montévil \& G. Longo. (2016). "Theoretical Principles for Biology: Organization.” Progress in Biophysics and Molecular Biology 122 (1): 24-35. 10.1016/j.pbiomolbio.2016.07.005.

Noble, D. (2006). The music of life. Oxford: Oxford U. P.

Noble, D. (2008). "Claude Bernard, the First Systems Biologist \& the Future of Physiology." Experimental Physiology 93 (1): 16-26. 10.1113/expphysiol.2007.038695.

Nykter, M., N. D. Price, M. Aldana, S. A. Ramsey, S. A. Kauffman, L. E. Hood, O. Yli-Harja \& I. Shmulevich. (2008). "Gene Expression Dynamics in the Macrophage Exhibit Criticality." Proceedings of the National Academy of Sciences 105 (6): (1897). 10.1073/pnas.0711525105.

Perazzo, C. A., E. A. Fernandez, D. R. Chialvo \& P. Willshaw. (2000). "Large Scale-Invariant Fluctuations in Normal Blood Cell Counts: A Sign of Criticality?" Fractals 8 (3): 279-83. 10.1142/S0218348X00000329.

Pikkujamsa, S. M., T. H. Makikallio, L. B. Sourander, I. J. Raiha, P. Puukka, J. Skytta, C.-K. Peng, A. L. Goldberger \& H. V. Huikuri. (1999). "Cardiac Interbeat Interval Dynamics from Childhood to Senescence: Comparison of Conventional and New Measures Based on Fractals and Chaos Theory." Circulation 100 (4): 393. 10.1161/01.CIR.100.4.393.

Sarti, A., G. Citti \& D. Piotrowski. (2019). "Differential heterogenesis and the emergence of semiotic function.” Semiotica. https://hal.archives-ouvertes.fr/hal-02123626.

Shmulevich, I., S. A. Kauffman \& M. Aldana. (2005). "Eukaryotic Cells Are Dynamically Ordered or Critical but Not Chaotic." Proceedings of the National Academy of Sciences 102 (38): 13439-44. 10.1073/pnas.0506771102.

Silverman, M. (2008). Quantum Superposition. Springer Verlag.

Sole, R. V., S. C. Manrubia, M. Benton, S. A. Kauffman \& P. Bak. (1999). "Criticality and Scaling in Evolutionary Ecology." Trends in Ecololgy \& Evolution 14 (4): 156-60. 10.1016/S01695347(98)01518-3.

Sonnenschein, C. \& A. M. Soto. (1999). The society of cells: cancer and control of cell proliferation. New York: Springer Verlag.

Soto, A. M., C. Sonnenschein \& P.-A. Miquel. (2008). "On Physicalism and Downward Causation in Developmental and Cancer Biology." Acta Biotheoretica 56 (4): 257-74. 10.1007/s10441-008-9052-y. 
Soto, A.M, Giuseppe L., D. Noble, N. Perret, M. Montévil, C. Sonnenschein, M. Mossio, et al. (2016). "From the Century of the Genome to the Century of the Organism: New Theoretical Approaches." Progress in Biophysics and Molecular Biology, Special Issue, 1-82.

Suzuki, M., K. Kaneko \& S. Takesue. (1982). "Critical Slowing down in Stochastic Processes. I." Progress of Theoretical Physics 67 (6): 1756-75. 10.1143/PTP.67.1756.

Toulouse, G., P. Pfeuty \& G. Barton. (1977). Introduction to the renormalization group and to critical phenomena. London: Wiley.

Tredicce, J. R., G. L. Lippi, P. Mandel, B. Charasse, A. Chevalier \& B. Picque. (2004). "Critical Slowing down at a Bifurcation." American Journal of Physics 72: 799. 10.1119/1.1688783.

Valverde, S., S. Ohse, M. Turalska, B.J. West \& J. Garcia-Ojalvo. (2015). "Structural Determinants of Criticality in Biological Networks." Frontiers in Physiology 6: 127.

Wagner, C. D., B. Nafz \& P. B. Persson. (1996). "Chaos in Blood Pressure Control." Cardiovascular Research 31 (3): 380. 10.1016/S0008-6363(96)00007.

Weibel, E. R. (1994). "Design of biological organisms and fractal geometry." In Fractals in Biology and Medicine. Vol. 1. Springer Verlag.

Werner, G. (2007). "Metastability, Criticality and Phase Transitions in Brain and Its Models." Biosystems 90 (2): 496-508. 10.1016/j.biosystems.2006.12.001.

West, G. B., J. H. Brown \& B. J. Enquist. (1997). “A General Model for the Origin of Allometric Scaling 
